\title{
A Virtual Laboratory Based on Full-Field Crystal Plasticity Simulation to Characterize the Multiscale Mechanical Properties of AHSS
}

Hongyue Ma

Shanghai Jiao Tong University

Yangqi Li

Shanghai Jiao Tong University

Haiming Zhang ( $\square$ hm.zhang@sjtu.edu.cn )

Shanghai Jiao Tong University

Qian Li

Institute of Shanghai Aircraft Design \& Research of Commercial Aircraft Corporation of China

Fei Chen

Shanghai Jiao Tong University

Zhenshan Cui

Shanghai Jiao Tong University

\section{Research Article}

Keywords: Crystal plasticity, Yield function, AHSS, Anisotropy, Virtual laboratory

Posted Date: August 5th, 2021

DOl: https://doi.org/10.21203/rs.3.rs-757841/v1

License: (9) (i) This work is licensed under a Creative Commons Attribution 4.0 International License.

Read Full License

Version of Record: A version of this preprint was published at Scientific Reports on March 23rd, 2022. See the published version at https://doi.org/10.1038/s41598-022-09045-8. 


\title{
A virtual laboratory based on full-field crystal plasticity simulation to characterize the multiscale mechanical properties of AHSS
}

Hongyue Ma ${ }^{1,2}$, Yangqi $\mathrm{Li}^{1,2}$, Haiming Zhang ${ }^{1,2 *}$, Qian $\mathrm{Li}^{3}$, Fei Chen ${ }^{1,2}$, Zhenshan Cui ${ }^{1,2}$

1. School of Materials Science and Engineering, Shanghai Jiao Tong University, 800 Dongchuan Road, Shanghai 200240, China

2. Institute of Forming Technology \& Equipment, Shanghai Jiao Tong University, 1954 Huashan Road, Shanghai, 200030, PR China

3. Institute of Shanghai Aircraft Design \& Research of Commercial Aircraft Corporation of China, Jinke Road, Shanghai, 201210, PR China

\begin{abstract}
In this work, we proposed a virtual laboratory based on full-field crystal plasticity simulation to track plastic anisotropy and to calibrate yield functions for multi-phase metals. The virtual laboratory, minimally, only requires easily accessible EBSD data for constructing the high-resolved microstructural representative volume element and macroscopic flow stress data for identifying the micromechanical parameters of constituent phases. An inverse simulation method based on global optimization scheme was developed for parameters identification, and a nonlinear least-squares method was employed to calibrate the yield functions. Various mechanical tests of an advanced high strengthening steel (DP980) sheet under different loading conditions were conducted to validate the virtual laboratory. Three well-known yield functions, the quadratic Hill48, Yld91, and Yld2004-18p, were selected as the validation benchmarks. All the studied functions, calibrated by numerous stress points under arbitrary loading conditions, successfully captured both the deformation and strength anisotropies. Furthermore, the full-field CP modeling well correlates the microscopic deformation mechanism and plastic heterogeneity to the macro-mechanical behavior of the sheet. The proposed virtual laboratory, which is readily extended with physically based CP model, could be a versatile tool to explore and predict the mechanical property and plastic anisotropy of advanced multi-phase metals.
\end{abstract}

Keywords: Crystal plasticity, Yield function, AHSS, Anisotropy, Virtual laboratory 


\section{Introduction}

During the past decades, advanced lightweight structural metals and alloys, represented with advanced high strengthen steels (AHSSs), are increasingly used to meet the demands for energy conservation and environmental protection ${ }^{1,2}$. The superior mechanical properties and high strength-to-weight ratio of AHSSs are primarily attributed to their synergetic enhancing mechanisms of strength and plasticity through the sophisticated controlling of the microstructure-property relations ${ }^{3}$. The complex microstructure, typically consisting of multiple phases over a wide range of length scales, renders the rather different macro-/micro-mechanical responses and plasticity behaviors. Specifically, plastic anisotropy of deformation and strength is one of the most concerns in the community of sheet metal forming ${ }^{4}$.

Describing the plastic anisotropy of metal sheets is a long-term research focus and challenge; in particular, phenomenological yield functions, which define a convex yield surface to separate the pure-elastic and elastoplastic deformation states and specify the direction of plastic flow with an associated or non-associated flow law, are widely employed to predict the macroscopic plastic anisotropy of rate-independent metals for their user-friendly implementation and high computational efficiency. The classic quadratic yield function Hill48 ${ }^{5}$, for instance, is the tacit yield criterion in most commercial FEM solvers for orthotropic materials; even though this criterion is short of simultaneously capturing deformation anisotropy (mostly measured with $r$-values, i.e., Lanford coefficients) and strength anisotropy ${ }^{6}$. With the emerging of progressive metals and alloys, especially the materials with multi-phase constituent or lowsymmetric crystal structure, more advanced non-quadratic yield functions have been receiving great attentions. Hosford ${ }^{7}$ proposed an isotropic yield criterion without shear stress on the basis of polycrystalline calculation and further extended to the planar anisotropy ${ }^{8}$. Whereafter, improved yield functions ${ }^{9-14}$ were introduced to more reasonably describe the anisotropy of metals based on the linear transformations of stress tensor. Furthermore, Barlat et al. ${ }^{12}$ and Aretz and Barlat ${ }^{15}$ introduced two advanced yield functions for generally stress state, namely Yld2004-18p and Yld2004- 
$27 p$, which require at most 18 and 27 material parameters to describe plastic anisotropy. These yield functions are mostly featured with lots of material parameters and growing alliance with crystallographic texture ${ }^{9,12}$.

The increased number of parameters causes the tough calibration of the advanced yield functions; the necessary mechanical experiments are time-consuming and sometimes impossible to complete, such as probing the out-of-plane properties of material sheets. Besides, the phenomenological yield functions are insufficient of physical mechanism and do not consider the microstructural heterogeneity; this weakens their efficient in predicting the plastic anisotropy of metal sheets with multiphase microstructure, of which the plastic heterogeneity and stress/strain partitions among microstructures and phases should not be ignored ${ }^{16,17}$.

Apart from the phenomenological yield criteria, micromechanical models and simulation tools based on crystal plasticity (CP) theory play a vital role in exploring the plastic anisotropy of metals and calibrating yield functions. For instance, Barlat et al. 12 used a mean-field visco-plastic self-consistent (VPSC) polycrystalline model to evaluate the out-of-plane mechanical properties of 2029-T3 Al-Li alloy sheet under different deformation models; combined with experimental results, the complete parameters of the Yld2004-18p yield function were identified. Plunkeet et al. ${ }^{18}$ fitted the material parameters of the CPB05 yield function by using the VPSC model in zirconium.

Recently, full-field CP modeling, either employs a finite element method (CPFEM) $19,20,21$ or a spectral method (CPSM) ${ }^{22,23}$ based on fast Fourier transformation as the solve of boundary-value problems, has received growing interest in characterizing the anisotropic behavior of polycrystalline materials. The full-field CP modeling, taking both grain microstructure and crystallographic texture as input, can guarantee the stress equilibrium and strain compatibility at grain boundaries and has the advantage over the mean-field ones of considering the authentic multiphase/polycrystalline microstructures and of describing the stress/strain partitions among different phases and grains $^{23,24}$. Zhang et al. ${ }^{25}$ employed a variety of CP based modeling approaches including CPFEM to investigate the anisotropic behavior of AA1050 aluminum and to 
calibrate the anisotropic parameters of the advanced Yld2004-18p function. Zhang et al. ${ }^{23}$ proposed a virtual laboratory (VL) based on high-resolution CPSM to predict the yield loci and plastic anisotropy of the annealed and cold-rolled AA3104 aluminum alloy sheets. The material parameters of the Yld91, Yld2000-2D, Yld2004-18p, and Yld2004-27p yield function were determined through a large number of yield stress data under arbitrary deformation paths. Liu et al. ${ }^{26}$ used the CPSM to determine a 3D phenomenological yield function with both in and out-of-plane mechanical anisotropy for modelling sheet metal forming of strongly textured AA3104-H19 and AA2024-T3. Han et al. ${ }^{27}$ employed CPSM combined with FEM simulations to dynamically calibrate the Yld2000-2D and Yld1004-18p yield functions for AA2090-T3 at different deformation stage, and FEM simulations were used to track the boundary conditions during the deep drawing process. These hierarchical modelling approaches combine the merits of both the time-saving of phenomenological yield functions for the engineering applications and the accuracy of micromechanical CP models.

However, most of the works mentioned above are focus on the single-phase metals and alloys. This work aims to introduce a full-field CP based VL which is capable of characterizing the multiscale mechanical properties of multiphase materials (using DP980 AHSS as an example), including macroscopic mechanical anisotropy and grainlevel heterogeneities. Comprehensive experiments were performed for the CP modeling setup and the validation of simulation results. Then a global optimum inverse-fitting procedure was developed to obtain the $\mathrm{CP}$ constitutive parameters for the constituent phases of the DP980 sheet. Virtual tests were carried out to obtain adequate yield stress points, from which the exemplified yield functions, i.e., Hill-48, Yld91, and Yld2004$18 \mathrm{p}$, were calibrated and the anisotropy of the DP980 sheet was explored. In the end, the correlation between the grain-level plasticity heterogeneity with the macromechanical property of the sheet were investigated. 


\section{Theory}

Finite strain CP theory. For the sake of completeness, the adopted phenomenological CP theory is briefly presented; more details can be found in Zhang et al. ${ }^{28}$. The CP model was implemented in a finite strain framework based upon the classical multiplication decomposition of deformation gradient as follows,

$$
\mathbf{F}=\mathbf{F}^{\mathbf{e}} \mathbf{F}^{\mathbf{p}}
$$

where $\mathbf{F}^{\mathbf{p}}$ describes the plastic slip on slip planes and $\mathbf{F}^{\mathbf{e}}$ describes the elastic stretching and the rotation of lattice. The plastic velocity gradient is expressed as,

$$
\mathbf{L}^{\mathrm{p}}=\sum_{\alpha} \dot{\gamma}^{\alpha} \mathbf{S}^{\alpha}, \text { with } \mathbf{S}^{\alpha}=\mathbf{m}^{\alpha} \otimes \mathbf{n}^{\alpha}
$$

where $\dot{\gamma}^{\alpha}$ is the slip rate of the $\alpha$-th slip system, and $\mathbf{S}^{\alpha}$ is the Schmid tensor. $\mathbf{m}^{\alpha}$ and $\mathbf{n}^{\alpha}$ denote the slip direction and the normal of the $\alpha$-th slip system. The slip rate $\dot{\gamma}^{\alpha}$ is described by a phenomenological plastic flow law as follows,

$$
\dot{\gamma}^{\alpha}=\dot{\gamma}_{0}\left|\frac{\tau^{\alpha}}{g^{\alpha}}\right|^{1 / m} \operatorname{sign}\left(\tau^{\alpha}\right), \text { with } \tau^{\alpha}=\left(\mathbf{F}^{\mathrm{eT}} \mathbf{F}^{\mathrm{e}} \cdot \mathbf{T}^{\mathrm{e}}\right): \mathbf{S}^{\alpha}
$$

where $\dot{\gamma}_{0}$ is the reference shear strain rate, $\tau^{\alpha}$ the resolved shear stress acting on the slip system, and $\mathbf{T}^{\mathrm{e}}$ the second-order Piola-Kirchhoff stress tensor. $m$ represents the strain rate sensitivity and $g^{\alpha}$ is the slip resistance which increases asymptotically from the initial value $g_{0}$ to the saturation value $g_{\mathrm{s}}$. To represent the work hardening behavior of crystalline materials, the evolution of $g^{\alpha}$ is formulated by a rate-form hardening model as follows ${ }^{29}$ :

$$
\dot{g}^{\alpha}=\sum_{\beta} h_{\alpha \beta} \dot{\gamma}^{\beta}, \text { with } h_{\alpha \beta}=h_{0}\left[q+(1-q) \delta^{\alpha \beta}\right]\left|1-\frac{g^{\beta}}{g_{\infty}}\right|^{a}
$$

where $h_{0}, g_{\infty}$, and $a$ are material parameters representing the reference selfhardening coefficient, the saturation value of the slip resistance, and the hardening exponent, respectively. Besides, the latent hardening parameter $q$ is routinely assumed to 1.0 for coplanar slip systems and 1.4 for non-coplanar slip systems.

With the assumption of hypo-elasticity of most metals, $\mathbf{T}^{\mathrm{e}}$ can be calculated from the elastic Green strain tensor $\mathbf{E}_{\mathrm{e}}$ as

$$
\mathbf{T}^{\mathrm{e}}=\mathbb{C}: \mathbf{E}_{\mathrm{e}} \text { with } \mathbf{E}_{\mathrm{e}}=\frac{\mathbf{1}}{\mathbf{2}}\left(\mathbf{F}^{\mathrm{eT}} \mathbf{F}^{\mathrm{e}}-\mathbf{I}\right)
$$

where $\mathbb{C}$ is the elasticity tensor; for materials with a cubic crystal structure, $\mathbb{C}$ is 
specified by three parameters, i.e., $C_{11}, C_{12}$, and $C_{44}$.

The above CP based constitutive model in conjunction with an open-source spectral solver DAMASK ${ }^{30}$ enable the VL to carry out a large number of virtual experiments with low computational cost ${ }^{23}$.

Modeling setup for full-field CP simulations. The geometrical model used for the full-field CP simulations and virtual tests, i.e., the multiphase microstructure representative volume element (RVE), was constructed via the open-source code DREAM.3D with the processed EBSD data as input. The cubic RVE, as shown in Fig.1, contains the essential microstructural characteristics of the DP980, including the phase volume fractions, the size distributions, and ODFs; it contains 100 elements (the Fourier grids with size of $0.5 \mu \mathrm{m}$ ) in each direction, and has 872 orientations ("grains") in the ferrite phase and 4193 orientations ("islands") in the martensite, respectively. Periodic boundary conditions were applied on the RVE to perform the simulations. In order to overcome the difficulty of obtaining different stress states in physical experiments, the method of rotating Euler angles of grain orientation is used instead of alternating the load directions ${ }^{22}$. Following the Bunge convention of Euler angles, the grain orientation for in-plane rotation from RD to TD at angle $\theta$ is $\left\{\varphi_{1}-\theta, \Phi, \varphi_{2}\right\}$ and for out-of-plane rotation from $\mathrm{RD}$ to normal direction (ND) at $\theta$ is $\left\{\varphi_{1}-\theta, \Phi+\right.$ $\left.90^{\circ}, \varphi_{2}\right\}$. In this way, the same RVE and PBCs were used for the full-field CP simulations, and a large number of random stress states were simulated for calibrating the phenomenological yield functions.
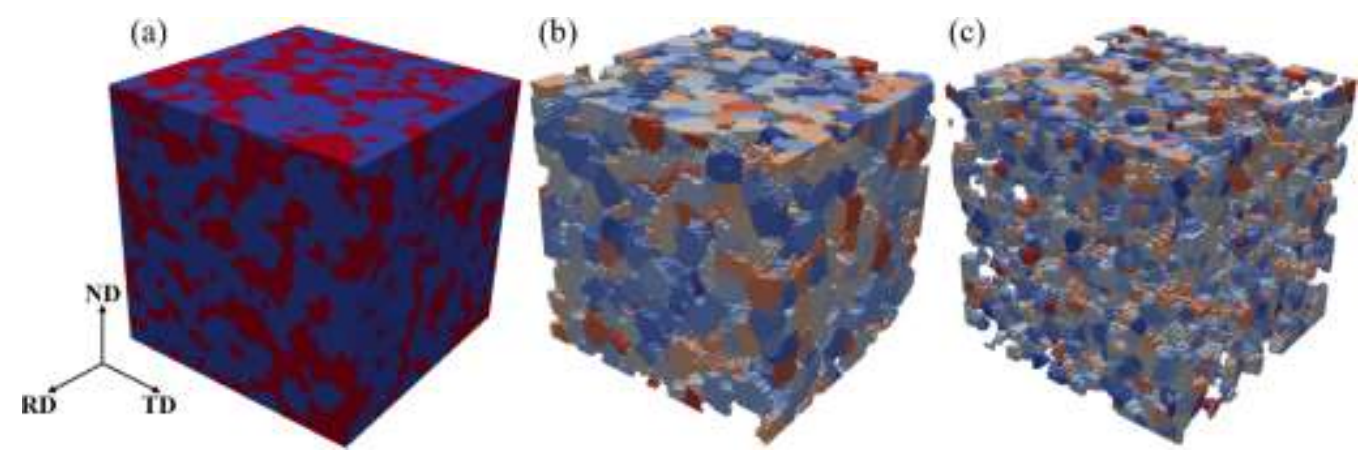

Figure 1. (a) The high-resolved dual-phase RVE (DP-RVE) used for CP simulations, with red represents martensite and blue represents ferrite; IPF color of the separated (b) ferrite and (c) martensite phases. 
Parameters identification for the CP constitutive model. Dual-phase steels have a composite microstructure consisting of relatively strong martensite particles dispersed in the softer and ductile ferrite matrix. Both the ferrite and the martensite phases have a BCC crystal structure (herein we ignore that slight distortion of the martensite's structure from the BCC structure) with the most easily activated slip systems at room temperature of $\{\overline{1} 10\}\langle 111\rangle$ and $\{\overline{2} 11\}\langle 111\rangle$ and each slip system has its own set of parameters. Thus, there are a lot of material parameters need to be identified. First of all, the elastic constants $C_{11}, C_{12}$, and $C_{44}$, the reference slip rates $\dot{\gamma}_{0}$, and the rate sensitivity coefficients $m$ are assumed to be identical for the two slip systems in each phase, and their value were routinely documented ${ }^{32}$. The four parameters, $g_{0}, g_{\infty}, h_{0}$, and $a$, which describe the work hardening behavior of slip systems in each phase were identified by an in-house inverse simulation procedure according to the uniaxial tensile data in RD and TD. The initial and saturation slip resistances $g_{0}$ and $g_{\infty}$ of system $\{\overline{1} 10\}\langle 111\rangle$ were predetermined to be smaller than those of $\{\overline{2} 11\}\langle 111\rangle$.

The inverse method for parameters identification consists of a global optimization platform and the full-field CP simulations. The particle swarm optimization (PSO) toolbox of MATLAB ${ }^{\circledR}$ was employed to identify the global optimum material parameters for the ferrite and martensite phases. In the beginning, a suitable bound of the parameters are provided for the inverse fitting platform; then the platform generates different guesses for the parameters, launches numerous full-field CP simulations of uniaxial tension parallelly, and finally extracts the predicted the true stress-strain data. The obtained true stress-strain data were compared with the experiment ones to evaluate the root-mean-square deviations (RMSD) $R_{\mathrm{c}}$ of objective functions in the current generation. Then the PSO toolbox introduced a new generation with optimized parameter sets. The fitting process will be iterated until the minimum $R_{\mathrm{c}}$ is below the prescribe limit. Fig. 2 shows the flow chart of the inverse fitting of the CP constitutive parameters. The objective function of the PSO optimization procedure is given as,

$$
R_{\mathrm{c}}=\sum_{i=1}^{n} w_{i}\left(\left(\sigma_{i}^{\operatorname{sim}}(x)-\sigma_{i}^{\mathrm{exp}}\right) / \sigma_{i}^{\mathrm{exp}}\right)^{2}
$$

where $x=\left[x_{1}, x_{2}, \ldots, x_{n}\right]$ are the parameters to be identified. $\sigma_{i}^{\text {sim }}$ and $\sigma_{i}^{\text {exp }}$ are the 
simulated and experimental stresses at the same strain, respectively, and $i$ denotes the corresponding data in different directions, i.e., RD and TD. $w_{i}$ is the weighting factor which is set to one in this work.

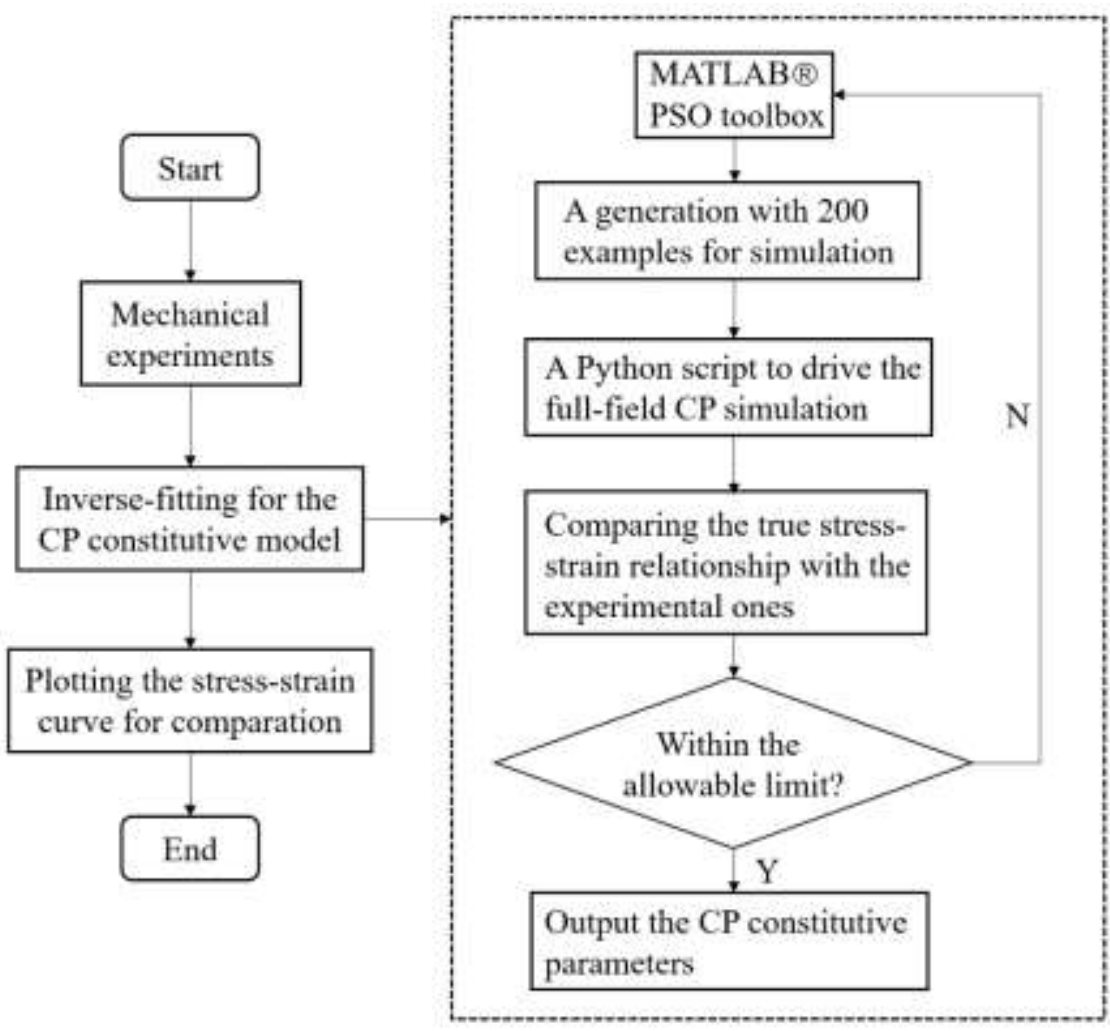

Figure 2. The flow chart of the in-house inverse method for parameters identification of the $\mathrm{CP}$ constitutive model.

Table 1 lists all the CP constitutive parameters used in this work, and Fig.3 shows the comparison between the experimental and the CP simulated flow stress curves of uniaxial tensions in both RD and TD. For $g_{0}$, the values of two slip systems of $\{\overline{1} 10\}\langle 111\rangle$ and $\{\overline{2} 11\}\langle 111\rangle$ in martensite are $4 \sim 5$ times higher than those in ferrite; for $g_{\infty}$, the values of $\{\overline{1} 10\}\langle 111\rangle$ and $\{\overline{2} 11\}\langle 111\rangle$ in martensite are $3 \sim 4$ and $1.5 \sim 2.5$ times higher than those in ferrite, respectively. 

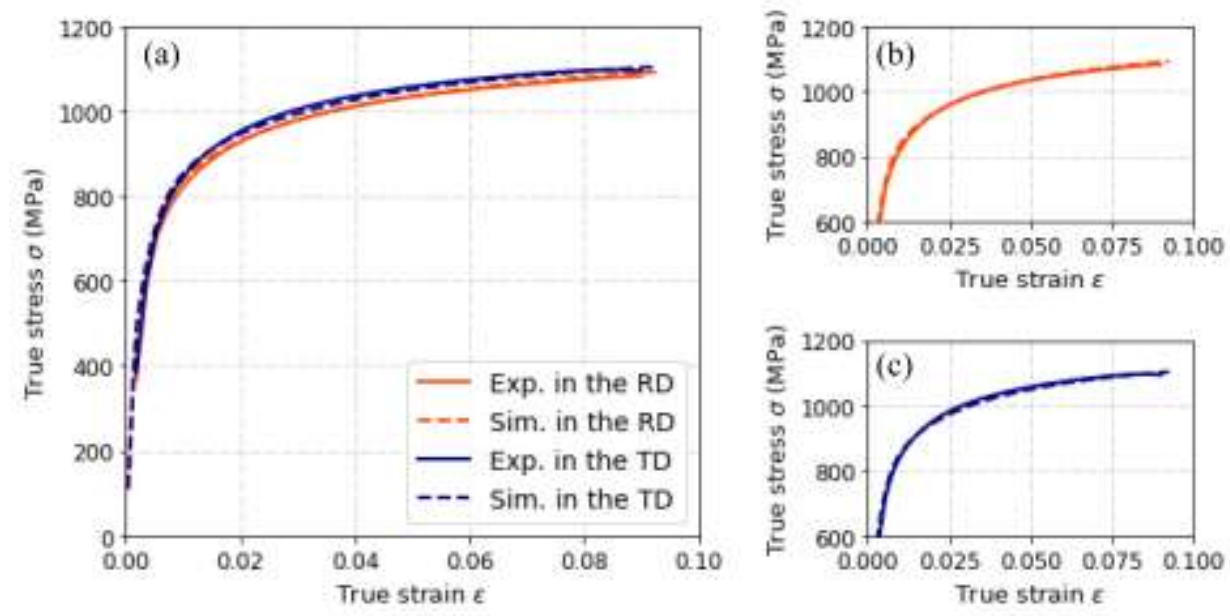

Figure 3. (a) Comparison of the flow stress curves predicted the calibrated CP model with the experimental ones along RD and TD. The partial magnification of results in $\mathrm{RD}$ is shown in (b) and in TD is shown in (c).

Table 1. Material parameters adjusted to the mechanical properties of both ferrite and martensite phases. The parameters of initial and saturation slip resistance, reference self-hardening modulus, and hardening exponent are determined by an in-house inverse-fitting procedure based on the experimental data; other parameters were routinely documented and referred to the literature ${ }^{31}$.

\begin{tabular}{|c|c|c|c|c|c|}
\hline \multirow{3}{*}{ Property } & \multicolumn{4}{|c|}{ Value } & \multirow{3}{*}{ Unit } \\
\hline & \multicolumn{2}{|c|}{ Ferrite } & \multicolumn{2}{|c|}{ Martensite } & \\
\hline & $\{\overline{1} 10\}\langle 111\rangle$ & $\{\overline{2} 11\}\langle 111\rangle$ & $\{\overline{1} 10\}\langle 111\rangle$ & $\{\overline{2} 11\}\langle 111\rangle$ & \\
\hline$g_{0}$ & 180.7 & 216.9 & 800.0 & 941.7 & $\mathrm{MPa}$ \\
\hline$g_{\infty}$ & 305.9 & 441.6 & 980 & 980.0 & $\mathrm{MPa}$ \\
\hline$h_{0}$ & 1.8 & 1.9 & 1.0 & 1.0 & $\mathrm{GPa}$ \\
\hline$a$ & 1.05 & 1.40 & 2.18 & 1.05 & - \\
\hline$\dot{\gamma}_{0}$ & \multicolumn{2}{|c|}{0.001} & \multicolumn{2}{|c|}{0.001} & $\mathrm{~s}^{-1}$ \\
\hline$m$ & \multicolumn{2}{|c|}{0.02} & \multicolumn{2}{|c|}{0.02} & - \\
\hline$C_{11}$ & \multicolumn{2}{|c|}{233.3} & \multicolumn{2}{|c|}{417.4} & $\mathrm{GPa}$ \\
\hline$C_{12}$ & \multicolumn{2}{|c|}{135.5} & \multicolumn{2}{|c|}{242.4} & $\mathrm{GPa}$ \\
\hline$C_{44}$ & \multicolumn{2}{|c|}{118.0} & \multicolumn{2}{|c|}{211.1} & $\mathrm{GPa}$ \\
\hline
\end{tabular}

Virtual laboratory. The VL is developed with an automatic Python script; it launches an enough number of full-field CP simulations of the constructed RVE under arbitrary loading conditions via a random number generator, post-processes the simulation results, extracts the yield stress points (stress tensors), and then identifies the yield functions. As the illustrative benchmarks, three well-known yield functions, i.e., the quadratic Hill48 ${ }^{5}$, the non-quadratic Yld91 ${ }^{9}$ and Yld2004-18p ${ }^{12}$, were calibrated to 
predict the anisotropy of the DP980 sheet.

The well-known nonlinear least-squares method (NLSM) fitting was adopted to identify the coefficients of the studied yield functions, and the NLSM problem is solved by using the bounded Levenberg-Marquardt optimization algorithm. This algorithm, with the analytical Jacobian matrix, provides enough robustness to find the solution of strong-nonlinear issues, even though the initial guess starts far off the final minimum. The objective function $O(\beta)$ is defined as follows.

$$
O(\beta)=\sum_{i=1}^{N}\left(\frac{\phi\left(\sigma^{i}, \beta_{j}\right)}{\sigma_{y}}-1\right)^{2}
$$

The effective stress corresponding to the considered yield functions is denoted as $\phi\left(\sigma^{i}, \beta\right)$, where $\sigma^{i}(i=1,2, \ldots, N)$ are the set of stress tensors of the corresponded virtual tests at the same plastic work per unit volume (shorten as the unit plastic work in the following content), and $\beta_{j}=\beta_{1}, \beta_{2}, \ldots, \beta_{M}$ are parameters of the yield functions to be calibrated. The optimal $\left\{\beta_{j}\right\}$ is to minimize the objective function $O(\beta)$. All the stress tensors were assigned with the same weight of unit in this study; however, different weights can be endowed to realize strong dependence of certain stress states. To stabilize and to accelerate the solving process of the objective function $O(\beta)$, the analytical Jacobian matrix was derived for the yield functions; the form of the Jacobian matrix is written as follows,

$$
J_{i j}=\frac{1}{\sigma_{y}} \frac{\partial \phi\left(\sigma^{i}, \beta\right)}{\partial \beta_{j}}, \text { with } 1 \leq i \leq N \text { and } 1 \leq j \leq M
$$

with $N$ and $M$ denote the number of stress points and the number of material parameters, respectively. For complex yield functions, e.g., Yld2004-18p, the chain rule was employed to get the Jacobian matrix.

The fitting quality is measured via RMSD $\left(R_{\mathrm{y}}\right)$ of the residual as follows,

$$
R_{\mathrm{y}}=\sqrt{\frac{1}{n} \sum_{n=1}^{N}\left(\frac{\phi\left(\sigma^{i}, \widehat{\beta}_{j}\right)}{\sigma_{y}}-1\right)^{2}}
$$

where $\hat{\beta}_{j}$ is the determined fitting parameters set for $\beta_{j}$.

Apart from the VL which is used to generate random yield points, a full-field CP virtual tests of uniaxial tension at a $7.5^{\circ}$ interval from RD to TD were also executed 
to predict the in-plane anisotropies of the DP980 sheet. The same RVE as the VL was used. Finally, $r$-values (the Lankford coefficients) and normalized yield stresses were extracted from the results of virtual tests.

\section{Results and discussion}

Microstructure. The studied material is a commercial cold-rolled and annealing DP980 steel sheet with thickness of $1.2 \mathrm{~mm}$ provided by Baosteel. The chemical composition of the steel is summarized in Supplementary Table 1.

Fig.4 displays the microstructure of the material characterized on the rolling direction (RD) - transverse direction (TD) plane in the center of thickness. Fig. 4a is the orientation imaging map (OIM) obtained from EBSD data, and the OIM is colored with the [100] inverse pole figure (IPF). Based on the band contrast (BC) value as shown in Fig.4b, the original microstructure was separated into the individual ferrite and martensite phases ${ }^{17}$. Fig. $4 \mathrm{c}$ presents the orientation distribution function (ODF) maps reconstructed from the EBSD data of the two individual phases. Both two phases show the typical texture of $\mathrm{BCC}$ metals after cold-rolling and annealing operations, i.e., the $\gamma$-fiber consisting of $\{111\}\langle u v w\rangle$ orientations and the $\alpha$-fiber consisting of $\{h k l\}\langle 110\rangle$. The dominant texture components in the ferrite phase, however, are different from those in the martensite phase. For the ferrite, the intensity of the $\alpha$-fiber is quite smaller than the $\gamma$-fiber, and the $\gamma$-fiber indeed concentrates at the texture component of $\{110\}\langle 1 \overline{2} 1\rangle$; for the martensite, on the contrary, the intensity of the $\alpha$ fiber is comparable with the $\gamma$-fiber, and the $\alpha$-fiber concentrates at $\{001\}\langle 110\rangle$ and the $\gamma$-fiber at $\{110\}\langle 1 \overline{2} 1\rangle$. It suggests a more enough sufficient annealing of the ferrite phase, as the annealing process of cold-rolled BCC materials strengthens the $\gamma$-fiber and reduces the intensity of $\alpha$-fiber ${ }^{22}$. 


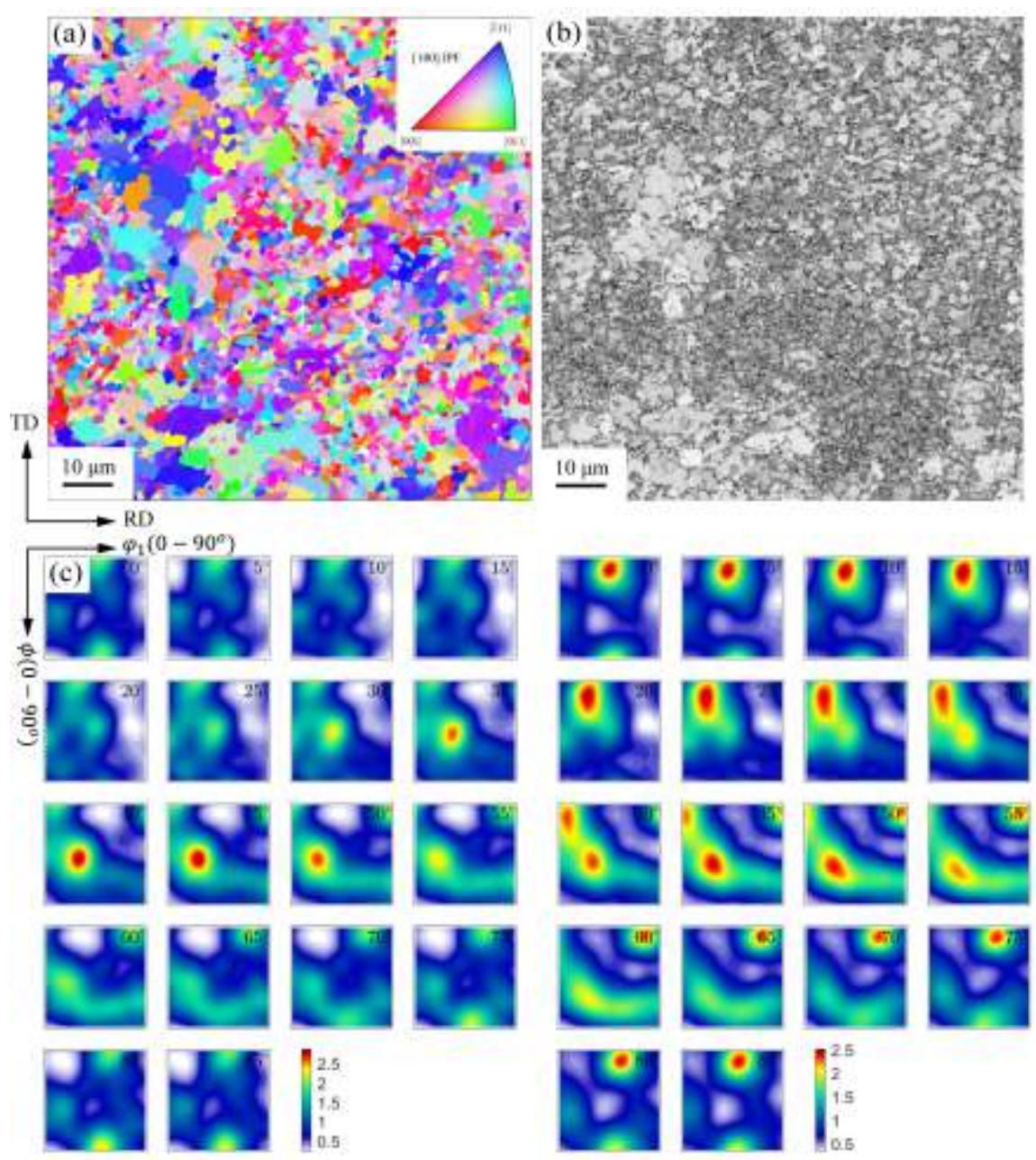

Figure 4. The initial microstructure of the as-received DP980 sheet characterized on the RD-TD plane at the center in thickness. (a) the EBSD orientation imaging map (OIM); (b) the band contrast (BC) map; (c) and (d) are the orientation density function (ODF) maps reconstructed from the EBSD data of ferrite and martensite, respectiveily. The ODFs were sectioned (constant $\varphi_{2}$ sections, from $0^{\circ}$ to $85^{\circ}$ in $5^{\circ}$ steps) through the reduced Euler space (with the Bunge convention) for the cubicorthorhombic symmetry.

The flow stress directionality and yield loci of the DP980 sheet. Fig.5 presents the flow stress curves of the DP980 sheet subjected to different loading conditions; in which Fig.5a shows the curves of the uniaxial tension in different directions, and Fig.5b the curves of pure shear and biaxial tension tests. The results exhibit the typical characteristic of cold-rolled dual-phase steels, i.e., no obvious yield point and stress plateau but continuous hardening. The von Mises equivalent stress of pure shear is obviously higher than the flow stresses of uniaxial tension in RD and biaxial tensions; 
while the biaxial flow stresses are a little smaller than that uniaxial one in the early deformation stage. With the increase of deformation, the equi-biaxial flow stress-strain curve can be higher than the uniaxial tension.
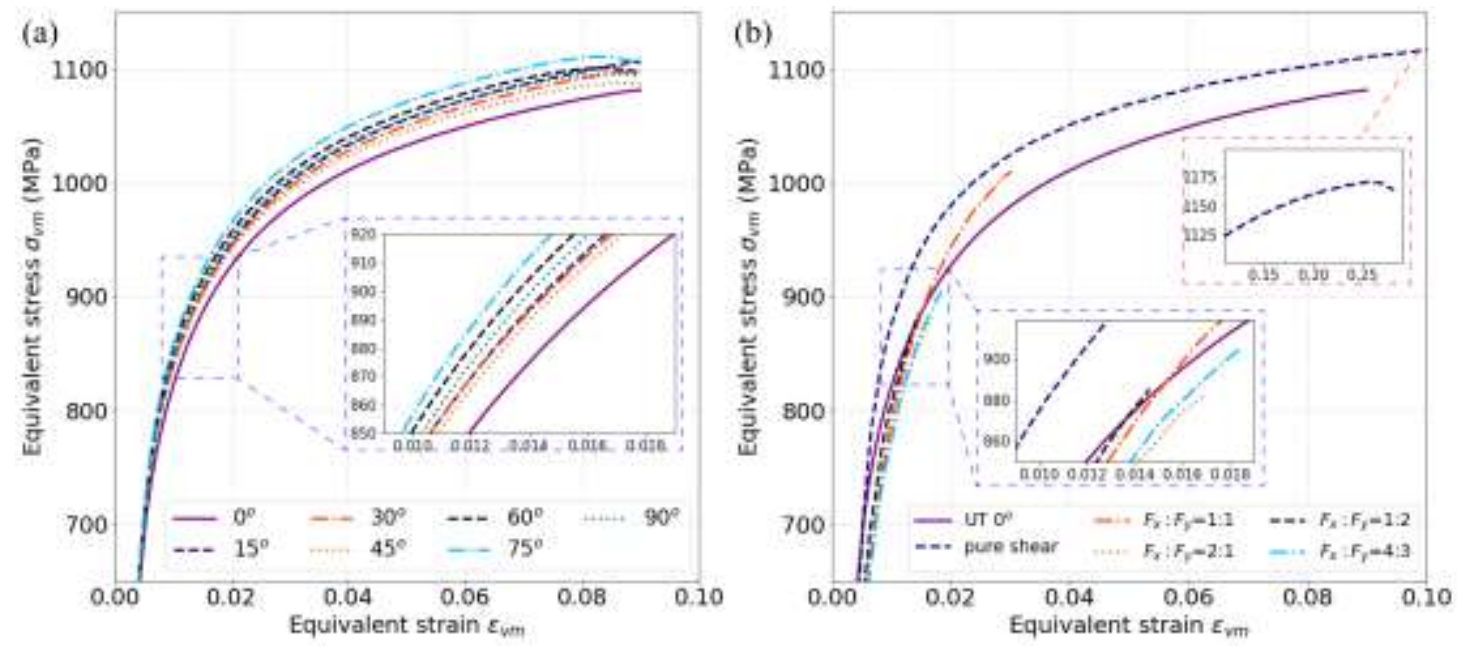

Figure 5. Flow stress curves of (a) uniaxial tension in different directions and (b) pure shear and biaxial tension under four different loading paths. The local amplifications were presented for convenient observation.

Fig. 6 presents the yield loci of the calibrated yield functions in $\frac{\sigma_{11}}{\bar{\sigma}}-\frac{\sigma_{22}}{\bar{\sigma}}$ plane, as well as the 60 different two-dimensional yield stress points randomly generated by the VL and the experimental results of biaxial tension with different stress ratios, pure shear, and uniaxial tension. For the studied DP980 sheet, the equi-biaxial equivalent stress is almost identical with the yield stress of uniaxial tension in RD but slightly higher than that in TD. All the simulated and experimental stress points were selected from the deformation stages with plastic work of $3 \mathrm{MPa}$ (Fig. 6a) and 8MPa (Fig. 6b). To verify the VL's capacity in predicting the plastic anisotropy of multi-phase materials, only the simulated 60 stress points at plastic work of $3 \mathrm{MPa}$ and $8 \mathrm{MPa}$ were used to identify the parameters of the yield functions. Table2 list the calibrated parameters. Different from the recommendations of Hosford ${ }^{7}$, which suggested $m=8$ for materials with FCC crystal structure and $m=6$ for BCC structure, the intentionally calibrated homogeneous exponent $m$ varies in the range of 5 8.

Table 2. The optimal parameters of the calibrated Hill48, Yld91, and Yld2004-18p yield functions for the DP980 sheet. 


\begin{tabular}{|c|c|c|c|c|c|c|c|c|}
\hline Yield & Plastic & \multicolumn{7}{|c|}{ Parameters } \\
\hline \multirow{3}{*}{ Hill48 } & & $F$ & $G$ & $H$ & $L$ & $M$ & $N$ & \\
\hline & $3 \mathrm{MPa}$ & 0.4809 & 0.5364 & 0.4822 & 1.5120 & 1.4272 & 1.5985 & \\
\hline & $8 \mathrm{MPa}$ & 0.4509 & 0.5029 & 0.4548 & 1.4035 & 1.3431 & 1.4958 & \\
\hline \multirow{3}{*}{ Yld91 } & 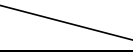 & $a$ & $b$ & c & $f$ & $g$ & $h$ & $m$ \\
\hline & $3 \mathrm{MPa}$ & 0.9852 & 1.0455 & 1.0030 & 0.9942 & 0.9848 & 1.0391 & 5.5246 \\
\hline & $8 \mathrm{MPa}$ & 0.9519 & 1.0153 & 0.9680 & 0.9608 & 0.9537 & 1.0070 & 5.7713 \\
\hline \multirow{9}{*}{$\begin{array}{c}\text { Yld2004- } \\
18 p\end{array}$} & - & $c_{12}$ & $c_{21}$ & $c_{23}$ & $c_{32}$ & $c_{31}$ & $c_{13}$ & $c_{44}$ \\
\hline & $3 \mathrm{MPa}$ & 0.9530 & 1.0290 & 0.9299 & 0.9317 & 1.1268 & 1.0910 & 0.9963 \\
\hline & $8 \mathrm{MPa}$ & 0.7263 & 1.1876 & 1.1016 & 0.8761 & 1.1304 & 0.9469 & 0.9776 \\
\hline & & $c_{55}$ & $c_{66}$ & $d_{12}$ & $d_{21}$ & $d_{23}$ & $d_{32}$ & $d_{31}$ \\
\hline & $3 \mathrm{MPa}$ & 0.0292 & 1.1575 & 0.9340 & 0.8417 & 0.9290 & 0.9620 & 0.6287 \\
\hline & $8 \mathrm{MPa}$ & 0.5326 & 1.2619 & 0.8406 & 0.9662 & 0.9852 & 0.8030 & 0.6843 \\
\hline & & $d_{13}$ & $d_{44}$ & $d_{55}$ & $d_{66}$ & $m$ & & \\
\hline & $3 \mathrm{MPa}$ & 0.9612 & 0.9853 & 1.4402 & 0.7265 & 7.2904 & & \\
\hline & $8 \mathrm{MPa}$ & 1.0006 & 0.9917 & 1.3642 & 0.5933 & 6.8343 & & \\
\hline
\end{tabular}

As shown in Fig.6, the yield loci depicted by the random stress points obtained from the VL agree well with those depicted by the experimental data, i.e., the VL successfully reproduced the yield loci of the DP980 sheet. All the yield functions, as calibrated with these randomly simulated stress points, are finely outline the yield loci of the sheet in comparison with either the experimental data or the VL produced ones. In particular, the yield loci predicted by the calibrated Yld91 and Yld2004-18p functions are quite coincident. While those predicted by the Hill48 yield function show some deviations from the others in the regions close to equi-biaxial tension and plane strain. This is reasonable that the quadratic Hill48 yield function is insufficient of capturing the large curvature change of yield locus in the region of biaxial tension. 

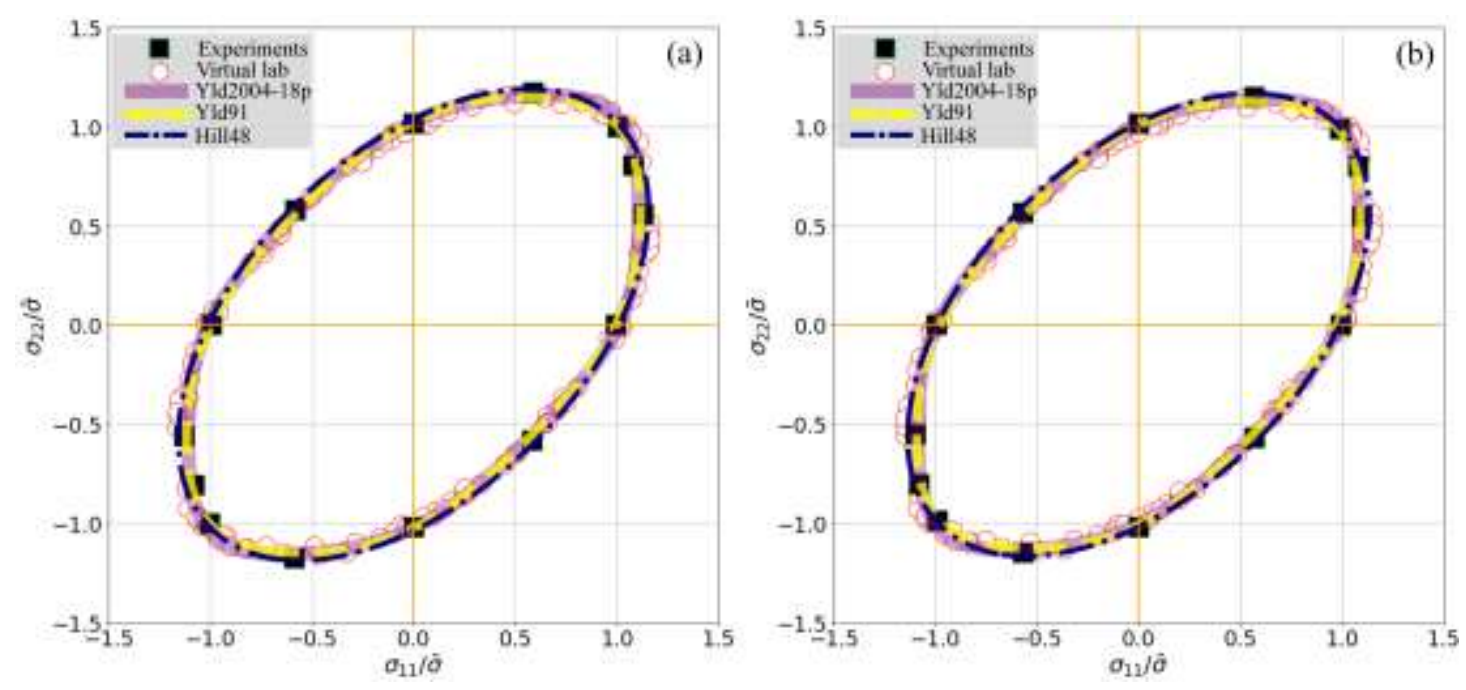

Figure 6. Yield loci $\left(\sigma_{12}=0\right)$ of the DP980 sheet outlined with experimental stress points (solid markers) of different loading conditions, enclosed by numerous stress points generated by the VL, and predicted by the calibrated yield function (lines); (a) the equvilent deformation stage with the unit plastic work of $3 \mathrm{MPa}$ and (b) $8 \mathrm{MPa} . \bar{\sigma}$ denotes the uniaxial tensile stress along the RD obtained from the virtual tests.

Fig.7 shows a direct comparison of yield loci of all the considered yield functions at various levels with equal normalized shear stress $\sigma_{12} / \bar{\sigma}$. The maximal $\sigma_{12} / \bar{\sigma}$ of the Yld2004-18p, Yld91, and quadratic Hill48 functions are 0.56009, 0.54134, and 0.56488 at the unit plastic work of $3 \mathrm{MPa}$, and $0.56057,0.55641$, and 0.58394 at the unit plastic work of $8 \mathrm{MPa}$, respectively. It can be seen from the results that the deviation among the yield functions increases with the ration of shear stress, and the difference is noticable in the case of $\sigma_{12} / \bar{\sigma}>0.5$.
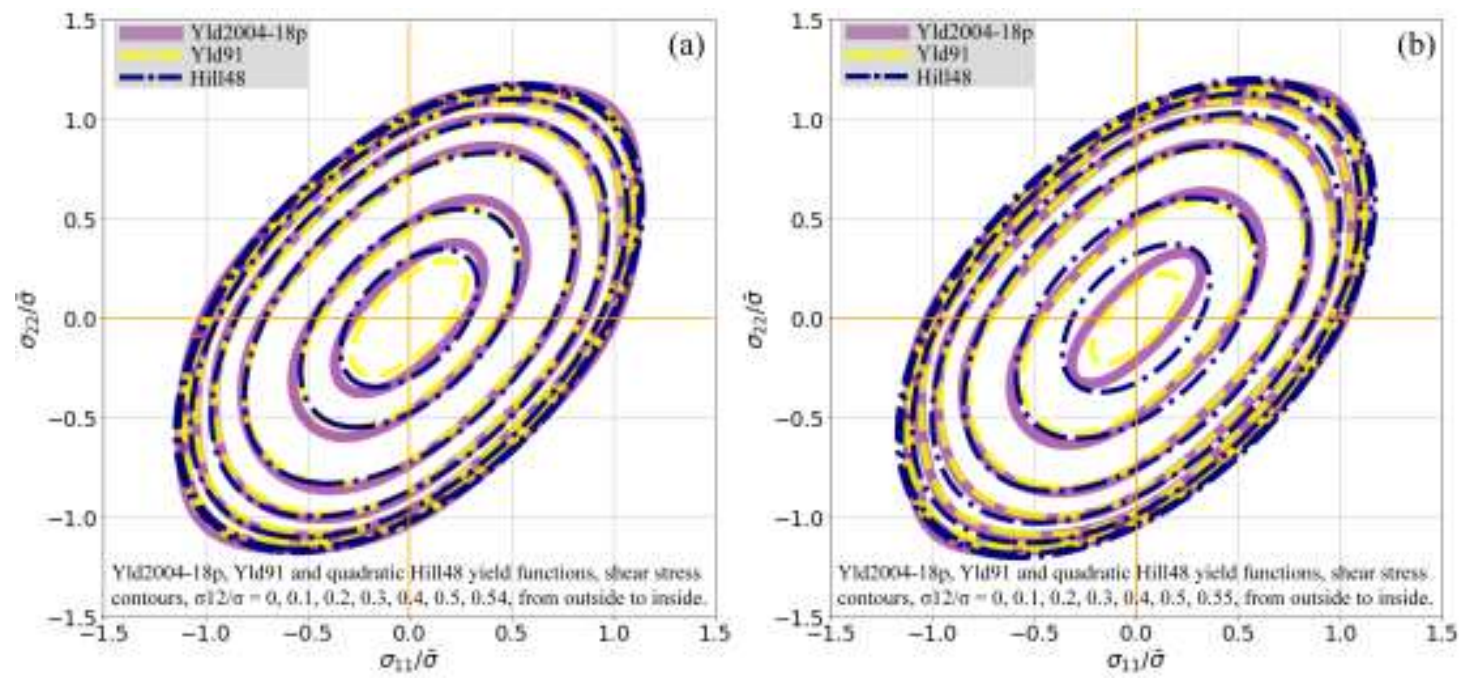

Figure 7. Tricomponent $\left(\sigma_{11}-\sigma_{22}-\sigma_{12}\right)$ yield loci with constant $\sigma_{12} / \bar{\sigma}$ contours 
predicted by the calibrated yield functions for the DP980 sheet; (a) the equvilent deformation stage with the unit plastic work of $3 \mathrm{MPa}$ and (b) $8 \mathrm{MPa} . \bar{\sigma}$ denotes the uniaxial tensile yield stress along the $\mathrm{RD}$ obtained from the virtual tests.

In-plane anisotropy of deformation and strength. For polycrystalline metal sheets, the variations of $r$-value and normalized yield stress $\left(Y_{\theta}\right)$ with uniaxial tensile directions are commonly employed to measure the in-plane anisotropy of deformation and strength. Fig. 8 presents the comparisons of the $r$-values predicted by the calibrated yield functions, obtained from the experiments, and from the virtual uniaxial tensions at deformation stages with unit plastic works of $3 \mathrm{MPa}$ (Fig. 8a) and 8MPa (Fig. 8b), respectively; and Fig. 9 presents the normalized yield stresses. Note that the experimental results at the unit plastic work of $60 \mathrm{MPa}$ were also provided for checking the variation of $r$-values with deformation.

As shown in Fig. 8, the $r$-values obtained from the experiments are fairly consistent with those predicted by the virtual tests or the calibrated yield functions. The DP980 sheet exhibits the typical deformation anisotropy of most cold-rolled and annealing BCC metal sheets, i.e., the maximum $r$-value in the diagonal direction (DD, i.e., the uniaxial tensile direction aligned $45^{\circ}$ with respect to the $\mathrm{RD}$ ) and the minimum in both RD and TD. The sheet has a small normal anisotropy with the average $\bar{r}\left(=\left(r_{0}+2 r_{15}+2 r_{30}+2 r_{45}+2 r_{60}+2 r_{75}+r_{90}\right) / 12\right)$ about 0.855 but a relatively strong in-plane anisotropy with $\Delta r / \bar{r}\left(\Delta r=2 r_{45}-r_{0}-r_{90}\right)$ about 0.279 . The virtual tests captured the variation tendency of $r$-values with the direction angle $\theta$ very well. Nevertheless, it is noticed that the $r$-values obtained by the virtual tests are a little higher than the experiments; this is might because that the potential $\langle 111\rangle$ pencilglide mechanism in BCC metals ${ }^{31}$, which does not prescribe a specific slip plane for the $\langle 111\rangle$ dislocation glide and thus enables a large number of alternative slip systems, was not considered the present CP constitutive model. In other words, a more advanced physically based CP might be able to yield an even better prediction of the plastic anisotropy for this type of AHSS. In addition, the experimental data evidence that the variation of $r$-values with deformation is not noticeable. At the deformation stages with the unit plastic work of $3 \mathrm{MPa}$ and $8 \mathrm{MPa}$, the $r$-values are hardly distinguishable 
in term of deformation; with the deformation increased to $60 \mathrm{MPa}$, a small decrease of $\bar{r}$ can be deduced from Fig. 8, i.e., the decrease of $r$-values in the directions near DD and the increase of $r$-values in both RD and TD.

The yield functions, as stated, calibrated with the stress points of virtual tests only, successfully captured the variation tendency of $r$-values. Especially, the advanced yield function Yld2004-18p predicts the variation of $r$-values in rather good agreements with both the virtual test and experimental results; other two yield functions, i.e., Hill48 and Yld91, slightly underestimate $\Delta r$ or the in-plane anisotropy. This is expectable as both have fewer material parameters for describing plastic anisotropy; in particular, the Yld91 criterion is a subset version of the Yld2004-18p as the former shares only one half (either the parameter matrix $\boldsymbol{C}$ or $\boldsymbol{D}$ illustrated in supplementary Eqs. 16-17) of parameters of the latter. The quadratic Hill48 yield function also predicts a faithful directionality of the $r$-values; this is, to some extent, attributed to the uncomplicated variation of the $r_{\theta}$ curves. The studied DP980 steel sheet, even though consists of a dual-phase microstructure, has only two stationary points in the $r_{\theta}$ curves; one locates in the RD/TD and another near the DD. The quadratic Hill48 criterion, as anticipated, is capable of capturing this phenomenon. In a word, although only stress points generated by the virtual tests were used to identify the anisotropy parameters of the yield functions, all the calibrated functions successfully capture the directionality and variation of $r$-values. The discrepancies among the predicted results of the functions can be also associated with the intrinsic characteristics of the functions.
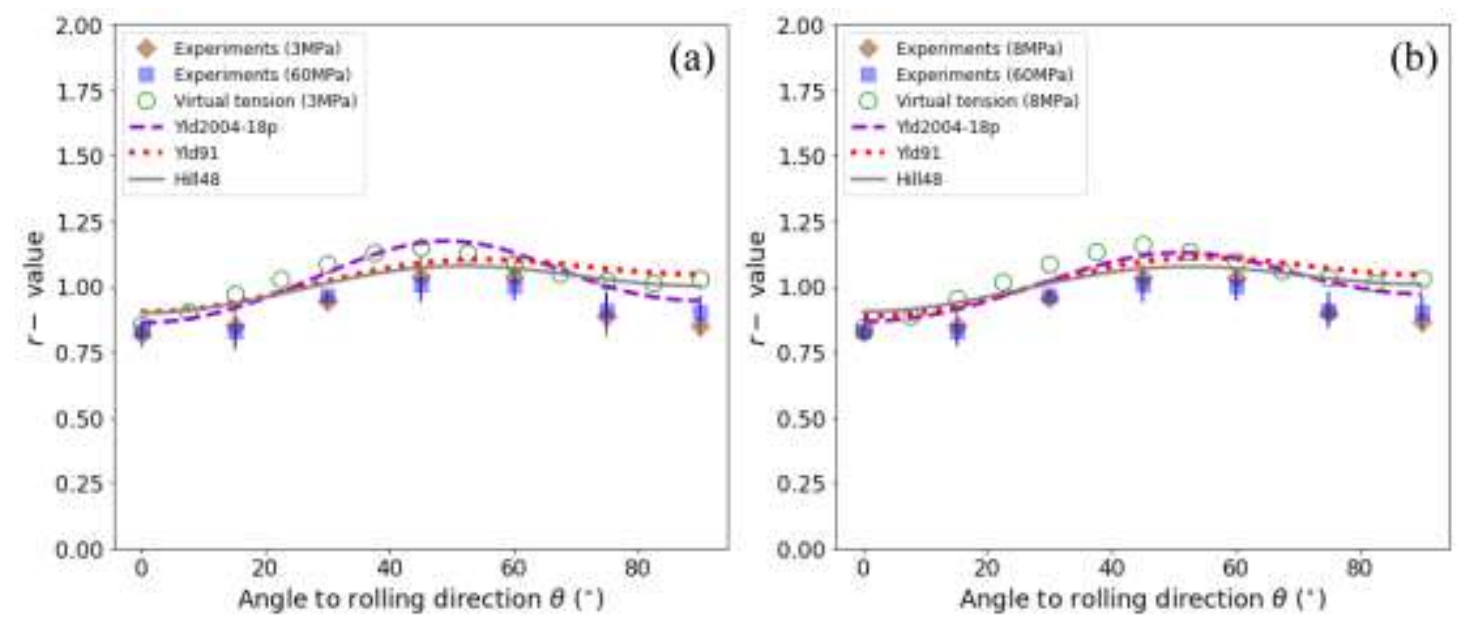
Figure 8. $r$-value versus $\theta$ of the DP980 sheet at deformation stages with the unit plastic work of (a) $3 \mathrm{MPa}$ and (b) $8 \mathrm{MPa}$. The lines are the predicted results of the calibrated yield functions. The diamonds and squares represent experimental values at a interval of $15^{\circ}$ from $\mathrm{RD}$ to $\mathrm{TD}$ and the open circles are the results obtained from virtual tests of uniaxial tension at a interval of $7.5^{\circ}$ from RD to TD.

As shown in Fig. 9, the DP980 sheet exhibits a relatively small strength anisotropy with the maximum variation of the normalized yield stress $Y_{\theta}$ below 5\%; this can be also demonstrated by the flow stress curves shown in Fig. 5. With the increase of deformation, the strength anisotropy was slightly weaken. Regardless of the rather small variation of $Y_{\theta}$, the stress points obtained by the virtual tests agree well with the experimental data except those in the directions with $\theta=30^{\circ}$ and $45^{\circ}$. All the calibrated yield functions predict a well consistent variation of $Y_{\theta}$ with the virtual test results.
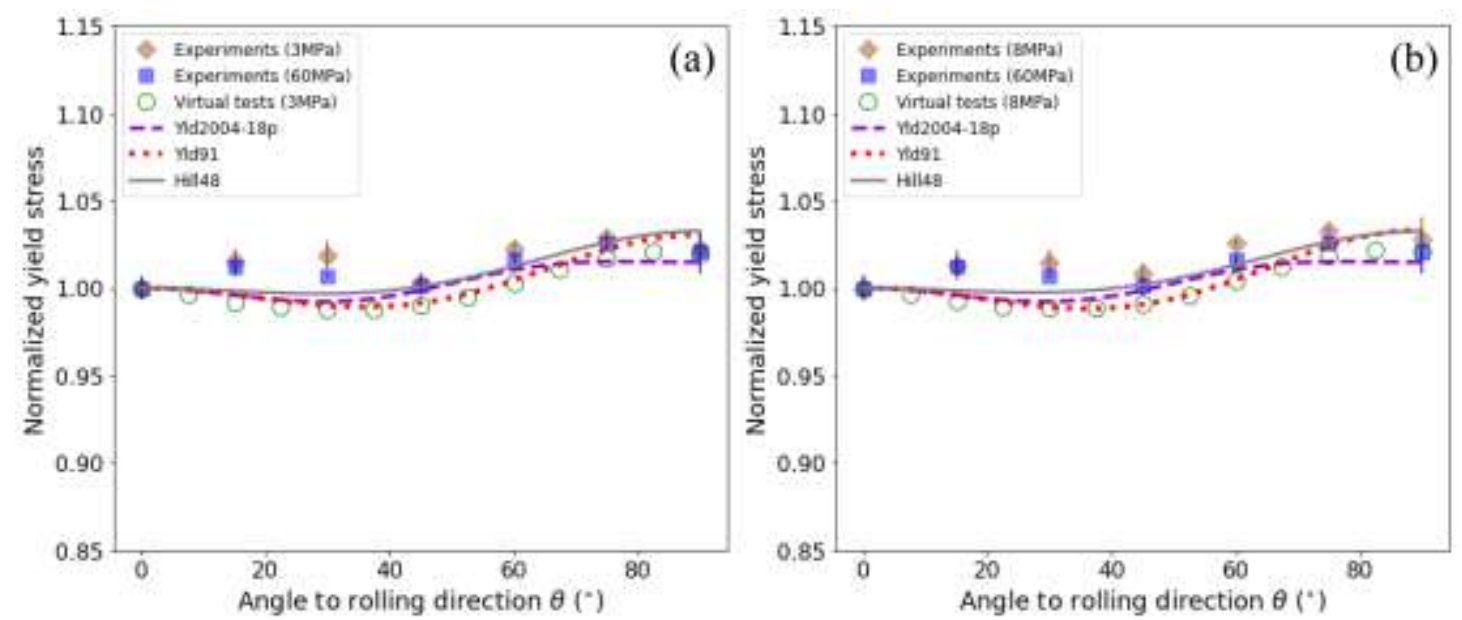

Figure 9. Normalized yield stress $Y_{\theta}$ versus $\theta$ of the the DP980 sheet at deformation stages with the unit plastic work of (a) $3 \mathrm{MPa}$ and (b) $8 \mathrm{MPa}$. The lines are the predicted results of the calibrated yield functions. The diamonds squares represent experimental values at a interval of $15^{\circ}$ from RD to TD and the open circles are the results obtained from virtual tests of uniaxial tension at a interval of $7.5^{\circ}$ from RD to TD.

It is noted that the Hill48 yield function, which is well-known for its incapacity of capturing the deformation anisotropy and the strength anisotropy simultaneously, gives a satisfactory prediction of the variations of both $r$-values and normalized yield stresses with tensile direction. Because of the cost and sometimes the restriction of physical experiments, the conventional calibration process of yield functions employs the equal-number experimental data with the parameter number to identify the 
anisotropic parameters. As stated by Zhang et al. ${ }^{22}$, this routine cannot yield an optimum set of parameters. The Hill48 function, for instance, may predict the deformation anisotropy well but the strength anisotropy poorly when calibrated by the experimental data $r$-values, and vice versa. By using the enough number (60 here) of stress points with arbitrary loading conditions, the studied yield functions, including the classical quadratic function and the advanced non-quadratic function, calibrated by the full-field CP based VL, can well predict the deformation and strength anisotropies simultaneously.

Plasticity heterogeneities at grain level. To study the role of individual phases in affecting the overall plasticity of the sheet, the microscopic distributions of tensile stress, tensile strain, and $r$-value were further investigated. Fig. 10 presents the contour maps of the deformed RVEs tensile tested in RD at the unit plastic work of $60 \mathrm{MPa}$; both the as-tested dual-phase RVE (DP-RVE) and the intentionally separated single-phase RVEs (F-RVE for ferrite and M-RVE for martensite) were provided for comparison. The black lines in the DP-RVE depict the phase boundaries. As anticipated, all the RVEs exhibit significantly inhomogeneous distributions of stress, strain, $r$-value. The martensite phase, for its higher strength, shows a larger stress level and a smaller strain level in comparison with the ferrite phase; while the distributions of $r$-value in the two phases are quite similar and strongly inhomogeneous. The contour maps of the DP-RVE manifest that strain hot-spots occur in some grains' interior and phase boundaries in the ferrite phase whereas stress hot-spots mainly in the martensitic grains.

To quantitatively understand the plasticity heterogeneity of the dual-phase microstructure, the histograms of frequency distribution of true stress, true strain, and $r$-value shown in Fig. 10 were further plotted in Fig.11. As shown in Fig. 11a, the strain frequency distribution of the ferrite essentially follows a Gaussian normal distribution with the median of $\sim 0.07$, whereas that of the martensite is more like a log distribution with the mode of $\sim 0.01$. It indicates that the ferrite phase, as expected, accommodates most applied deformation and that the deformation in the ferrite is much more homogeneous than that in the martensite. As $\mathrm{Li}$ et al. ${ }^{17}$ demonstrated, the 
statistical strain distribution of the single-phase material, either the ferrite or the martensite, should also follow the normal distribution; the log distribution of the martensite's strain is inferred because of the strongly non-uniform deformation partition between the phases. Because of the apparently different strain distributions in the individual phases, the DP-RVE (i.e., the actual DP980 sheet) exhibits a nonprobability distribution of strain, which suggests the statically non-uniform deformation of the steel sheet. On the contrary, the stress frequency distributions of both phases manifest as the typical Gaussian distribution, as shown in Fig. 11b. This, on the one hand, demonstrates the enough number of material points (Fourier grids) and grains (orientations) in the high-resolved RVE for representing the dual-phase microstructure, on the other hand, implies the statistically uniform stress distribution of the individual phases. While the standard deviation of the martensite is obviously larger than that of the ferrite, which implies a more uniform stress distribution in the ferrite. As anticipated, the median stress $(\sim 1400 \mathrm{MPa})$ of the martensite is much larger than that $(\sim 825 \mathrm{MPa})$ of the ferrite. Besides, unlike the strain frequency distribution, the stress distribution of the DP-RVE is also well bell-shaped.

The frequency distribution of $r$-value is rather different from those of strain and stress. For the martensite phase, as shown in Fig. 11c, there are more than $30 \%$ material points with $r$-value close to zero and $\sim 13 \%$ with $r$-value above 5.0 , and these values for the ferrite is $\sim 20 \%$ and $\sim 11 \%$ repectively. Apart from these two extreme sides, the $r$-value frequency distributions of the ferrite and martensite phases are greatly similar; both manifest a power-law distribution. All these features solidly imply a significant microscopic deformation anisotropy of the DP980 sheet, although its overal normal anisotropy shown in Fig. 8 is not very strong. Besides, Fig. 11c displays that the statistical $r$-value of the martensite is noticeably smaller than that of the ferrite. This phenomenon coincides with the initial crystallographic textures of the two phases as shown in Fig. 4, i.e., the martensite's texture contains a considerable intensity of $\alpha$-fible which weakens the $r$-value of materials; besides, the more inhomogeneous deformation in the martensite might also account for its smaller $r$-value. 


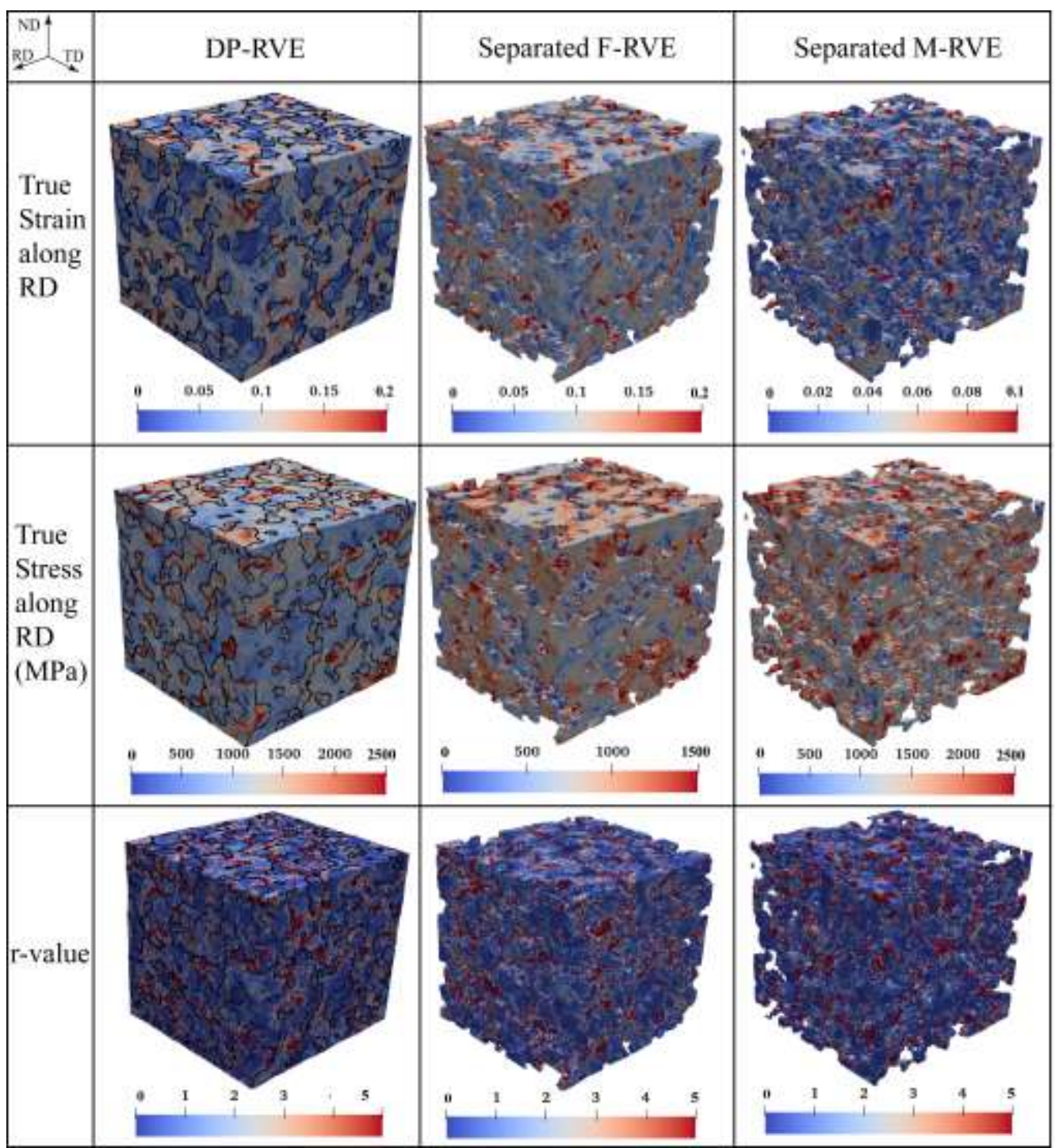

Figure 10. The contour maps of the true strain, true stress, and $r-$ value of the DPRVE, separated M-RVE and F-RVE along RD. The true stress and true strain correspond to the components in tensile direction.
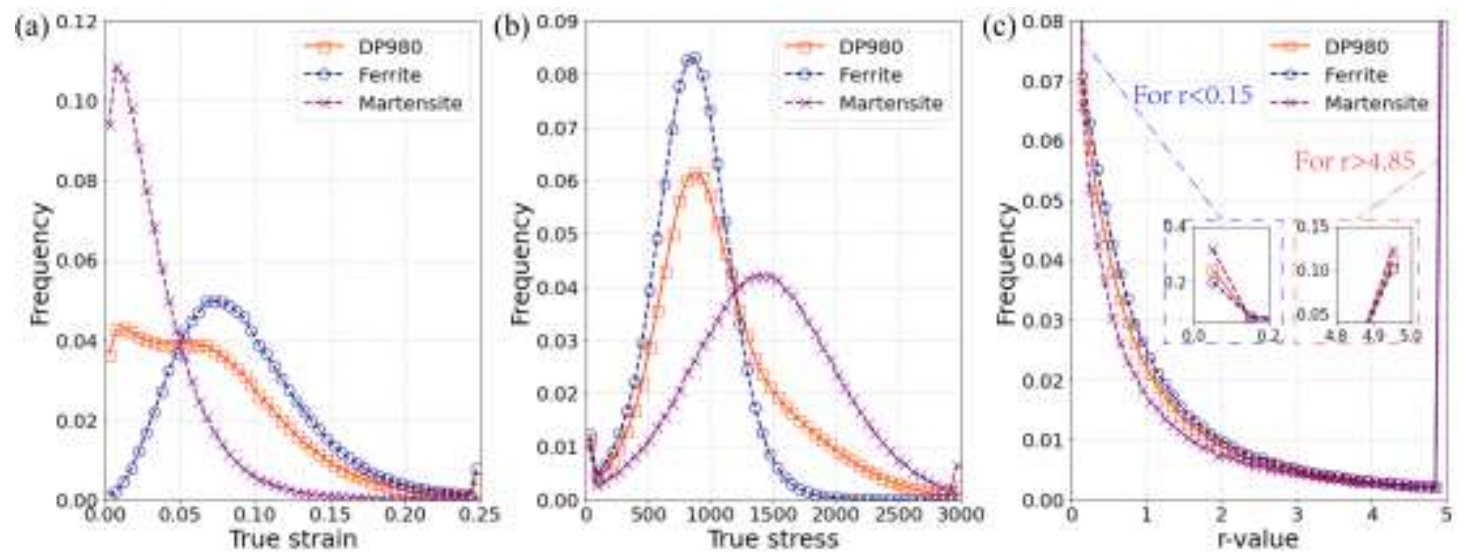

Figure 11 The histograms of frequency distribution of the true stress, true strain, and $r$-value of the DP-RVE, separated F-RVE and M-RVE. The RVE was subjected to 
uniaxial tension along; and the ture stress and ture strain corresponds to the components in tensile direction.

It is noted that RVEs tensile tested along other directions exhibit the similar results as those presented in Fig. 11 and Fig. 12. In summary, these results demonstrate that the $\mathrm{CP}$ based VL with the highly-resolved RVE correlates adequate micro-mechanisms and deformation heterogeneity of the dual-phase microstructure to the macroscopic plastic anisotropy of the DP980 sheet.

\section{Summary}

A virtual laboratory (VL) based on full-field crystal plasticity (CP) modeling was presented to investigate the mechanical anisotropy and to predict yield surfaces of multi-phase metals. The VL consists of four modules including a CP constitutive model, high-resolved representative volume element of multi-phase microstructure, an inverse method procedure based on global optimization for identifying the CP constitutive parameters of the constituent phases, and a local optimization scheme for calibrating yield functions with a large number of simulated stress points.

Elaborate mechanical experiments and microstructure characterization were carried out for modelling setup as well as validations of the CP parameters and of the calibrated yield functions. Both the yield loci generated by the VL and predicted by the calibrated yield functions agree well with the experiments, which were enveloped by yield stress points of uniaxial tensions, pure shear, and biaxial tensions. The deformation and strength anisotropies were finely captured by the VL and the calibrated yield functions. Especially the Hill48 yield function, which is generally incapable of capturing the $r$-value and the normalized yield stress $\left(Y_{\theta}\right)$ simultaneously, presents a satisfactory prediction of both $r$-value and $Y_{\theta}$. This is mainly attributed to the optimized parameters that were identified from the abundant stress points of arbitrary loading conditions generated by the VL.

Plasticity heterogeneities on grain level were investigated based on the dual-phase RVE, intentionally separated single-phase RVEs. It demonstrates that the CP based VL with highly-resolved RVEs correlates adequate micro-mechanisms and deformation 
heterogeneity of the multi-phase microstructure to the macroscopic plastic anisotropy of the material.

\section{Experiments}

Microstructural characterizations. The initial microstructure and texture of the asreceived sheet were characterized by the SEM electron-backscattered diffraction (EBSD) system, i.e., the VEGA 3 XMU (LaB6) field emission SEM (TESCAN) equipped with an Oxford/Nordlys EBSD detector. The SEM-EBSD system was operated at acceleration voltage of $20 \mathrm{kV}$ and working distance of $20 \mathrm{~mm}$; it scanned the area with size of $100 \mu \mathrm{m} \times 100 \mu \mathrm{m}$ and step size of $0.25 \mu \mathrm{m}$, i.e., collected 160,000 data points totally. The data was processed by the AZtec ${ }^{\circledR}$ system (Oxford Inst.) and reproduced with the open-source MATLAB ${ }^{\circledR}$ toolbox MTEX.

Mechanical tests. To obtain the parameters of the following CP constitutive model and to validate the yield functions determined by the full-field CP based VL, roomtemperature mechanical tests, including uniaxial tension, pure shear, and biaxial tension, were conducted for the as-received sheet. The geometric shapes and dimensions of these specimens are illustrated in Supplementary Fig.1a. The specimens were machined via electrical discharge machining; for the biaxial tensile specimens, seven slits were fabricated on each arm by laser cutting to reduce the geometric constraint on the deformation zone ${ }^{33}$.

Uniaxial tensile tests and pure shear tests were carried out quasi-statically on an electronic testing machine (Instron Model 8080 with load cell of 100kN capacity) equipped with a commercial digital image correlation (DIC) system (ARAMIS), as shown in Supplementary Fig.1b, at constant crosshead speeds of $3 \mathrm{~mm} / \mathrm{min}$ and 2 $\mathrm{mm} / \mathrm{min}$, respectively. Uniaxial tensile tests were carried out in the directions of every $15^{\circ}$ from $\mathrm{RD}$ to $\mathrm{TD}$, and five experiments were carried out for each direction to obtain the repeatable experimental data. The biaxial tensile tests were carried out along four loading paths (with stress ratios of $F_{x}: F_{y}=1: 1,2: 1,1: 2$, and $4: 4$ ) on biaxial tensile testing machine (MTS BIA5105) as shown in Supplementary Fig.1c at constant equivalent loading rate of $0.49 \mathrm{kN} / \mathrm{s}$. Two load cells were employed to measure the 
tensile force along RD and TD in real-time, respectively.

To facilitate the full-field strain measurements of the DIC system, all the specimens were uniformly sprayed with random speckle patterns prior to the tests. The three-dimensional position change of the speckles was photographed by dual highresolution cameras at a frequency of $2 \mathrm{~Hz}$; the strain field of the deformed specimens then was computed by the commercial DIC software GOM. The photographing was automatically synchronized with the load cell signal by the DIC data acquisition system.

\section{Reference}

1. Moon, J. et al. A new class of lightweight, stainless steels with ultra-high strength and large ductility. Sci. Rep. 10, 12140 (2020).

2. Sohn, S. S., Song, H., Kwak, J. H. \& Lee, S. Dramatic improvement of strain hardening and ductility to $95 \%$ in highly-deformable high-strength duplex lightweight steels. Sci. Rep. 7, 1927 (2017).

3. Raabe, D. et al. Current Challenges and Opportunities in Microstructure-Related Properties of Advanced High-Strength Steels. Metall. Mater. Trans. A 51, 5517-5586, (2020).

4. Liu, W. \& Chen, B. K. Sheet metal anisotropy and optimal non-round blank design in highspeed multi-step forming of AA3104-H19 aluminium alloy can body. Int. J. Adv. Manuf. Technol. 95, 4265-4277 (2018).

5. Hill, R. A Theory of the Yielding and Plastic Flow of Anisotropic Metals. Proc. R. Soc. A-Math. Phys. Eng. Sci. 193, 281-297 (1948).

6. Banabic, D. Sheet Metal Forming Processes: Constitutive Modelling and Numerical Simulation. Springer-Verlag Berlin Heidelberg (2010).

7. Hosford, W. F. A Generalized Isotropic Yield Criterion. J. Appl. Mech. 39, 607-609 (1972).

8. Barlat, F. \& Lian, K. Plastic behavior and stretchability of sheet metals. Part I: A yield function for orthotropic sheets under plane stress conditions. Int. J. Plast. 5, 51-66 (1989).

9. Barlat, F., Lege, D. J. \& Brem, J. C. A six-component yield function for anisotropic materials. Int. J. Plast. 7, 693-712 (1991).

10. Barlat, F., Maeda, Y. \& Chung, K. Yield function development for aluminum alloy sheets. J. Mech. Phys. Solids. 45, 1727-1763 (1997).

11. Barlat, F. et al. Plane stress yield function for aluminum alloy sheets—part 1: theory. Int. J. Plast. 19, 1297-1319 (2003).

12. Barlat, F. et al. Linear transfomation-based anisotropic yield functions. Int. J. Plast. 21, 1009-1039 (2005).

13. Karafillis, A. P. \& Boyce, M. C. A general anisotropic yield criterion using bounds and a transformation weighting tensor. J Mech Phys Solids 41, 1859-1886 (1993).

14. Banabic, D., Kuwabara, T., Balan, T., Comsa, D. S. \& Julean, D. Non-quadratic yield criterion for orthotropic sheet metals under plane-stress conditions. Int. J. Mech. Sci. 45, 
797-811 (2003).

15. Aretz, H. \& Barlat, F. New convex yield functions for orthotropic metal plasticity. Int. J. Non-Linear Mech. 51, 97-111 (2013).

16. Diehl, M. et al. Crystal plasticity study on stress and strain partitioning in a measured 3D dual phase steel microstructure. Phys. Mesomech. 20, 311-323 (2017).

17. Li, Q. et al. Study on the plastic anisotropy of advanced high strength steel sheet: Experiments and microstructure-based crystal plasticity modeling. Int. J. Mech .Sci. 176 (2020).

18. Plunkett, B., Lebensohn, R. A., Cazacu, O. \& Barlat, F. Anisotropic yield function of hexagonal materials taking into account texture development and anisotropic hardening. Acta. Mater. 54, 4159-4169 (2006).

19. Feather, W. G., Lim, H. \& Knezevic, M. A numerical study into element type and mesh resolution for crystal plasticity finite element modeling of explicit grain structures. Comput. Mech. 67, 33-55 (2020).

20. Han, F., Roters, F. \& Raabe, D. Microstructure-based multiscale modeling of large strain plastic deformation by coupling a full-field crystal plasticity-spectral solver with an implicit finite element solver. Int. J. Plast. 125, 97-117 (2020).

21. Wang, H. et al. A crystal plasticity FEM study of through-thickness deformation and texture in a $\{112\}<111>$ aluminium single crystal during accumulative rollbonding. Sci. Rep. 9, 3401 (2019).

22. Roters, F. et al. DAMASK: the Düsseldorf Advanced MAterial Simulation Kit for studying crystal plasticity using an FE based or a spectral numerical solver. Procedia IUTAM 3, 310 (2012).

23. Zhang, H., Diehl, M., Roters, F. \& Raabe, D. A virtual laboratory using high resolution crystal plasticity simulations to determine the initial yield surface for sheet metal forming operations. Int. J. Plast. 80, 111-138, (2016).

24. Zhang, H., Liu, J., Sui, D., Cui, Z. \& Fu, M. W. Study of microstructural grain and geometric size effects on plastic heterogeneities at grain-level by using crystal plasticity modeling with high-fidelity representative microstructures. Int. J. Plast. (2018).

25. Zhang, K. et al. Multi-level modelling of mechanical anisotropy of commercial pure aluminium plate: Crystal plasticity models, advanced yield functions and parameter identification. Int. J. Plast. 66, 3-30 (2015).

26. Liu, W., Chen, B. K., Pang, Y. \& Najafzadeh, A. A 3D phenomenological yield function with both in and out-of-plane mechanical anisotropy using full-field crystal plasticity spectral method for modelling sheet metal forming of strong textured aluminum alloy. Int. J. Solids. Struct. 193-194 (2020).

27. Han, F., Diehl, M., Roters, F. \& Raabe, D. Using spectral-based representative volume element crystal plasticity simulations to predict yield surface evolution during large scale forming simulations. J. Mater. Process. Technol. 277 (2020).

28. Zhang, H., Dong, X., Wang, Q. \& Zeng, Z. An effective semi-implicit integration scheme for rate dependent crystal plasticity using explicit finite element codes. Comput. Mater. Sci. 54, 208-218 (2012).

29. Kalidindi, S. R., Bronkhorst, C. A. \& Anand, L. Crystallographic texture evolution in bulk deformation processing of FCC metals. J. Mech. Phys. Solids. 40, 537-569 (1992). 
30. Roters, F. et al. DAMASK - The Düsseldorf Advanced Material Simulation Kit for modeling multi-physics crystal plasticity, thermal, and damage phenomena from the single crystal up to the component scale. Comput. Mater. Sci. 158, 420-478 (2019).

31. Tasan, C. C. et al. Integrated experimental-simulation analysis of stress and strain partitioning in multiphase alloys. Acta. Mater. 81, 386-400 (2014).

32. Hosford, W. F. Mechanical behavior of materials. Cambridge university press, 2010.

33. Min, J., Carsley, J. E., Lin, J., Wen, Y. \& Kuhlenkötter, B. A non-quadratic constitutive model under non-associated flow rule of sheet metals with anisotropic hardening: Modeling and experimental validation. Int. J. Mech. Sci. 119, 343-359 (2016).

\section{Acknowledgments}

The authors acknowledge the funding support from the National Natural Science Foundation of China with the projects of Nos. 52075329 and 51705317, as well as the supports from Shanghai Rising-Star Program (20QA1405300) and the Program of Shanghai Academic Research Leader (Grant No.19XD1401900).

\section{Credit author statement}

Hongyue Ma: Simulations, Data analysis, and Writing-Original draft preparation.

Yangqi Li: Experiments, Visualization, and Discussion

Haiming Zhang: Supervision, Methodology, Conceptualization, Writing - Review \& Editing, Data Curation, Funding acquisition.

Qian Li: Experiments and Data analysis.

Fei Chen: Discussion.

Zhenshan Cui: Discussion and Editing.

\section{Conflict of interest}

The authors declare no competing interests.

\section{Additional Information}

Supplementary information accompanies this paper at Supplementary $\underline{\text { Information.docx }}$ 


\section{Supplementary Files}

This is a list of supplementary files associated with this preprint. Click to download.

- SupplementaryInformation.docx 